\title{
Ionomer Cross-linking Immobilization of Catalyst Nanoparticles for High Performance Alkaline Membrane Fuel Cell
}

Xian Liang, ${ }^{\dagger}+$ Muhammad Aamir Shehzad, ${ }^{\dagger}$ Yuan Zhu, ${ }^{\dagger}$ Lianqin Wang, ${ }^{\S}$ Xiaolin Ge, ${ }^{\dagger}$ Jianjun Zhang, ${ }^{\dagger}$ Zhengjin Yang, ${ }^{\dagger}$ Liang $\mathrm{Wu}{ }^{*}{ }^{, \dagger}$ John Robert Varcoe, ${ }^{\S}$ and Tongwen $\mathrm{Xu}{ }^{*, \dagger}$

† CAS Key Laboratory of Soft Matter Chemistry, Collaborative Innovation Center of Chemistry for Energy Materials, School of Chemistry and Material Science, University of Science and Technology of China, Hefei 230026, P. R. China ¥ School of Chemistry and Material Engineering, Huainan Normal University, Huainan 232001, P. R. China $\S$ Department of Chemistry, The University of Surrey, Guildford, Surrey GU2 7XH, United Kingdom

These authors contributed equally. 
(a)

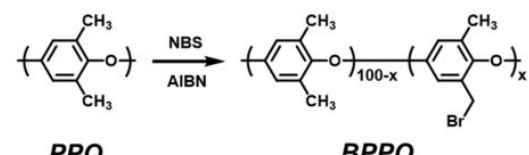

(b)

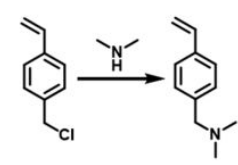

VBC VBN

(c)

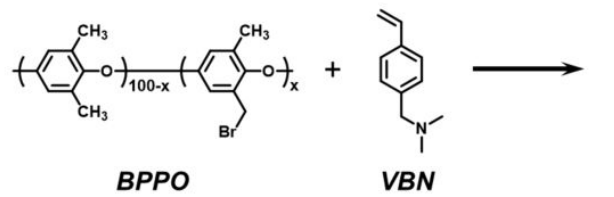

(d)

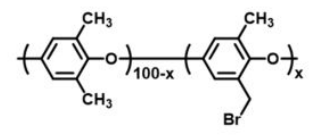

BPPO

(e)
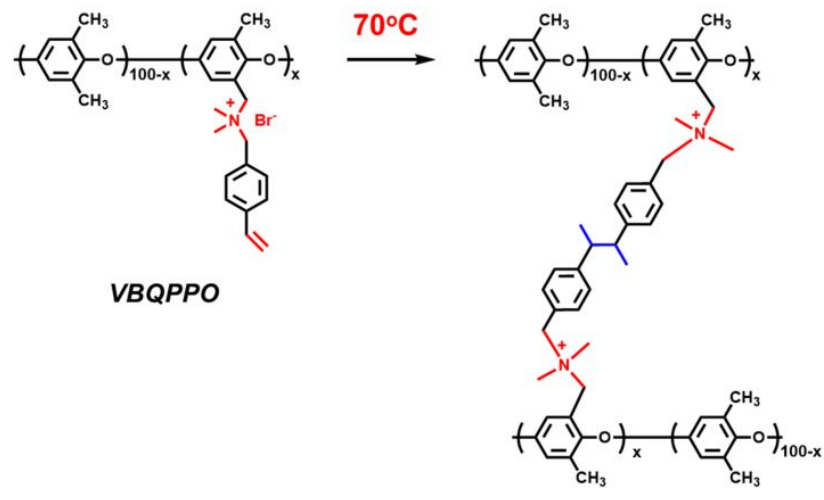

CBQPPO

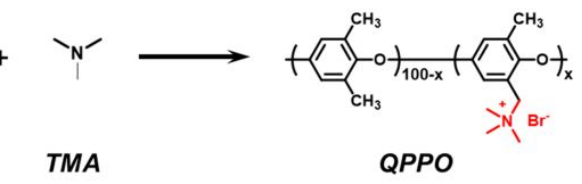

VBQPPO

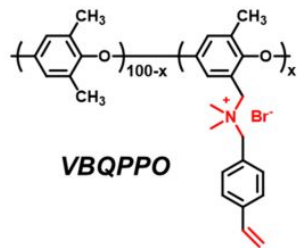

QPPO

Scheme S1. Synthesis of BPPO, VBN, VBQPPO, QPPO and CBQPPO. 
Table S1. Performance of recently reported AEIs or AEMs for $\mathrm{H}_{2} / \mathrm{O}_{2}$ alkaline membrane fuel cell.

\begin{tabular}{|c|c|c|c|c|c|c|c|c|c|}
\hline \multirow[t]{2}{*}{ AEIs or AEMs } & \multirow{2}{*}{$\begin{array}{c}\text { Peak Power } \\
\text { Density } \\
\left(\mathrm{W} \mathrm{cm}^{-2}\right)\end{array}$} & \multirow{2}{*}{$\begin{array}{c}\text { Cell Temp } \\
\left({ }^{\circ} \mathrm{C}\right)\end{array}$} & \multirow{2}{*}{$\begin{array}{c}\text { A/C Temp } \\
\left({ }^{\circ} \mathrm{C}\right)\end{array}$} & \multicolumn{2}{|c|}{$\begin{array}{c}\text { Back Pressure } \\
\text { (MPa) }\end{array}$} & \multicolumn{2}{|c|}{ Catalyst } & \multirow{2}{*}{$\begin{array}{c}\text { A/C Metal } \\
\text { Loading } \\
\mathrm{mg} \mathrm{cm}^{-2}\end{array}$} & \multirow[t]{2}{*}{ Literature } \\
\hline & & & & Anode & Cathode & Anode & Cathode & & \\
\hline CBQPPO (This Work) & 1.37 & 70 & $70 / 70$ & 0.1 & 0.1 & $\mathrm{PtRu} / \mathrm{C}$ & $\mathrm{Pt} / \mathrm{C}$ & $0.5 / 0.5$ & \\
\hline QAPPT & 1.45 & 80 & $80 / 80$ & 0.1 & 0.1 & $\mathrm{Pt} / \mathrm{C}$ & $\mathrm{Pt} / \mathrm{C}$ & $0.4 / 0.4$ & 1 \\
\hline aQAPS & 1.10 & 60 & 60 & 0.1 & 0.1 & $\mathrm{PtRu} / \mathrm{C}$ & $\mathrm{MCS} / \mathrm{C}$ & $0.4 / 0.58$ & 2 \\
\hline APEs & 1.92 & 80 & $80 / 80$ & 0.1 & 0.1 & $\mathrm{PtRu} / \mathrm{C}$ & $\mathrm{Pt} / \mathrm{C}$ & $0.4 / 0.4$ & 3 \\
\hline ETFE-VBTMAC & 1.35 & 70 & - & 0 & 0 & $\mathrm{PtRu} / \mathrm{C}$ & $\mathrm{CF} / \mathrm{C}$ & $0.07 / 2.4$ & 4 \\
\hline ETFE-VBCTMA & 1.40 & 60 & $54 / 57$ & 0 & 0 & $\mathrm{PtRu} / \mathrm{C}$ & $\mathrm{Pt} / \mathrm{C}$ & $0.67 / 0.53$ & 5 \\
\hline ETFE-BTMA & 1.00 & 70 & $40 / 42$ & 0.3 & 0.2 & $\mathrm{PdCeO}_{2} / \mathrm{C}$ & $\mathrm{PdCu} / \mathrm{C}$ & $0.42 / 0.58$ & 6 \\
\hline ETFE-BTMA & 1.90 & 60 & $45 / 46$ & 0 & 0 & $\mathrm{PtRu} / \mathrm{C}$ & $\mathrm{Pt} / \mathrm{C}$ & $0.71 / 1.07$ & 7 \\
\hline LDPE15 & 2.02 & 80 & $78 / 78$ & 0 & 0 & $\mathrm{PtRu} / \mathrm{C}$ & $\mathrm{Pt} / \mathrm{C}$ & $0.6 / 0.4$ & 8 \\
\hline LDPE & 1.45 & 80 & $80 / 80$ & 0 & 0 & $\mathrm{PtRu} / \mathrm{C}$ & $\mathrm{Pt} / \mathrm{C}$ & $0.6 / 0.4$ & 9 \\
\hline ETFE & 1.16 & 60 & $60 / 60$ & 0 & 0 & $\mathrm{PtRu} / \mathrm{C}$ & $\mathrm{Pt} / \mathrm{C}$ & $0.6 / 0.4$ & 10 \\
\hline HDPE & 2.55 & 80 & $78 / 78$ & 0 & 0 & $\mathrm{PtRu} / \mathrm{C}$ & $\mathrm{Pt} / \mathrm{C}$ & $0.6 / 0.4$ & 11 \\
\hline ETFE-MPRD & 1.43 & 60 & $60 / 60$ & 0 & 0 & $\mathrm{PtRu} / \mathrm{C}$ & $\mathrm{Pt} / \mathrm{C}$ & $0.6 / 0.4$ & 12 \\
\hline ETFE-VBC & 1.22 & 60 & - & 0 & 0 & $\mathrm{PtRu} / \mathrm{C}$ & $\mathrm{Pt} / \mathrm{C}$ & $0.6 / 0.4$ & 13 \\
\hline FLN-55 & 1.46 & 80 & $80 / 80$ & 0.285 & 0.285 & $\mathrm{PtRu} / \mathrm{C}$ & $\mathrm{Pt} / \mathrm{C}$ & $0.5 / 0.6$ & 14 \\
\hline Poly(norbornene) & 3.40 & 80 & $66 / 74$ & 0 & 0 & $\mathrm{PtRu} / \mathrm{C}$ & $\mathrm{Pt} / \mathrm{C}$ & $1.0 / 0.56$ & 15 \\
\hline
\end{tabular}


Table S2. Simulation electrical equivalent circuit (EEC) fits for the impedance spectra recorded with QPPO- and CBQPPO-ionomer-containing MEAs before and after the fuel cell durability tests. The measured (msd) EISNyquist data in Figure 3b was simulated (sim) with a best-fit equivalent electrical circuit model (standard error $\left.<0.5 \%, \chi^{2}<10^{-4}\right), \mathrm{LR}_{\mathrm{M}}\left(\mathrm{Q}_{\mathrm{MGI}} \mathrm{R}_{\mathrm{MGI}}\right)\left(\mathrm{Q}_{\text {cat }} \mathrm{R}_{\text {cat }}\left(\mathrm{Q}_{\mathrm{P}} \mathrm{R}_{\mathrm{P}}\right)\right.$ ), where contribution of the electrically conductive fuel cell components including electrodes was modeled by the inductance element (L) and $R_{M}$ represents the ohmic resistance of the membrane. The first circuit, $\left(Q_{M G I} R_{M G I}\right)$ attributes the charge-storage $(Q=$ constant phase element) and charge-transfer resistance (R) between the membrane-GDE interface (MGI). The second circuit, $\left(Q_{\text {cat }} R_{\text {cat }}\left(Q_{P} R_{P}\right)\right.$ ) represents the charge-storage and charge-transfer resistance values at the catalyst-polymer interface within the GDE. The $Q_{n}$ is the index value $\left(0<Q_{n}<1\right)$ of the constant phase element (Q).

\begin{tabular}{|c|c|c|c|c|c|c|c|c|c|c|c|c|c|}
\hline \multirow{2}{*}{$\begin{array}{c}\text { EEC } \\
\text { Parameters }\end{array}$} & \multirow{2}{*}{$\begin{array}{c}\text { Error } \\
(\%)\end{array}$} & \multirow{2}{*}{$\begin{array}{l}\text { Chi Sq. } \\
\text { value }\end{array}$} & \multirow[b]{2}{*}{ L (H) } & \multirow{2}{*}{$\underset{(\mathrm{m} \Omega)}{\mathrm{R}_{\mathrm{M}}}$} & \multicolumn{2}{|c|}{$\mathbf{Q}_{\text {MGI }}$} & \multirow{2}{*}{$\begin{array}{c}\mathbf{R}_{\mathrm{MGI}} \\
(\mathrm{m} \Omega)\end{array}$} & \multicolumn{2}{|c|}{$\mathbf{Q}_{\text {Cat }}$} & \multirow{2}{*}{$\begin{array}{c}R_{\text {Cat }} \\
(\mathrm{m} \Omega)\end{array}$} & \multicolumn{2}{|l|}{$\mathbf{Q}_{\mathbf{P}}$} & \multirow{2}{*}{$\begin{array}{c}R_{P} \\
(m \Omega)\end{array}$} \\
\hline & & & & & $\begin{array}{c}\mathbf{Y}_{\text {MGI }} \\
\left(\mathrm{S} \mathrm{cm}^{-2} \mathrm{~s}^{\mathrm{n}}\right) \\
\end{array}$ & $\mathbf{Q}_{\mathrm{n}-\mathrm{MGI}}$ & & $\begin{array}{c}\mathbf{Y}_{\text {Cat }} \\
\left(\mathrm{S} \mathrm{cm}^{-2} \mathrm{~S}^{\mathrm{n}}\right) \\
\end{array}$ & $\mathbf{Q}_{\text {n-Cat }}$ & & $\begin{array}{c}\mathbf{Y}_{\mathbf{P}} \\
\left(\mathrm{S} \mathrm{cm}^{-2} \mathrm{~S}^{\mathrm{n}}\right)\end{array}$ & $\mathbf{Q}_{\mathbf{n}-\mathbf{P}}$ & \\
\hline $\begin{array}{c}\text { QPPO@Pt/C } \\
\text { before }\end{array}$ & 0.471 & $2.22 \mathrm{E}-05$ & $1.38 \mathrm{E}-7$ & 48.7 & 0.712 & 0.762 & 28.4 & 0.047 & 0.655 & 97.3 & 0.406 & 0.851 & 27.7 \\
\hline $\begin{array}{l}\text { QPPO@Pt/C } \\
\text { after }\end{array}$ & 0.404 & $1.63 \mathrm{E}-05$ & $0.78 \mathrm{E}-7$ & 81.4 & 0.608 & 0.796 & 241 & 0.004 & 0.708 & 298 & 0.106 & 0.922 & 251 \\
\hline $\begin{array}{l}\text { CBQPPO@Pt/C } \\
\text { before }\end{array}$ & 0.389 & $1.52 \mathrm{E}-05$ & $1.22 \mathrm{E}-7$ & 42.9 & 0.695 & 0.806 & 26.7 & 0.073 & 0.629 & 21 & $4.679 \mathrm{E}-4$ & 0.801 & 51.8 \\
\hline $\begin{array}{l}\text { CBQPPO@Pt/C } \\
\text { after }\end{array}$ & 0.283 & $0.80 \mathrm{E}-05$ & $1.20 \mathrm{E}-7$ & 47.1 & 0.043 & 0.814 & 40.5 & 0.005 & 0.624 & 58.6 & $9.638 \mathrm{E}-5$ & 0.806 & 53.3 \\
\hline
\end{tabular}


Table S3. The total pore volume and BET surface area of QPPO@Pt/C-based MEAs and CBQPPO@Pt/C-based MEAs, before and after the durability test.

\begin{tabular}{ccc}
\hline Parameters & Total pore volume* $\mathbf{1 0}^{-\mathbf{2}} \mathbf{( c c / g )}$ & Surface Area $\left(\mathbf{m}^{\mathbf{2}} \mathbf{g} \mathbf{g}\right)$ \\
\hline QPPO@Pt/C_initial & 6.247 & 10.111 \\
QPPO@Pt/C_after 6.5h & 5.618 & 9.584 \\
CBQPPO@Pt/C_initial & 7.149 & 11.417 \\
CBQPPO@Pt/C_after 6.5h & 7.093 & 11.865 \\
\hline
\end{tabular}




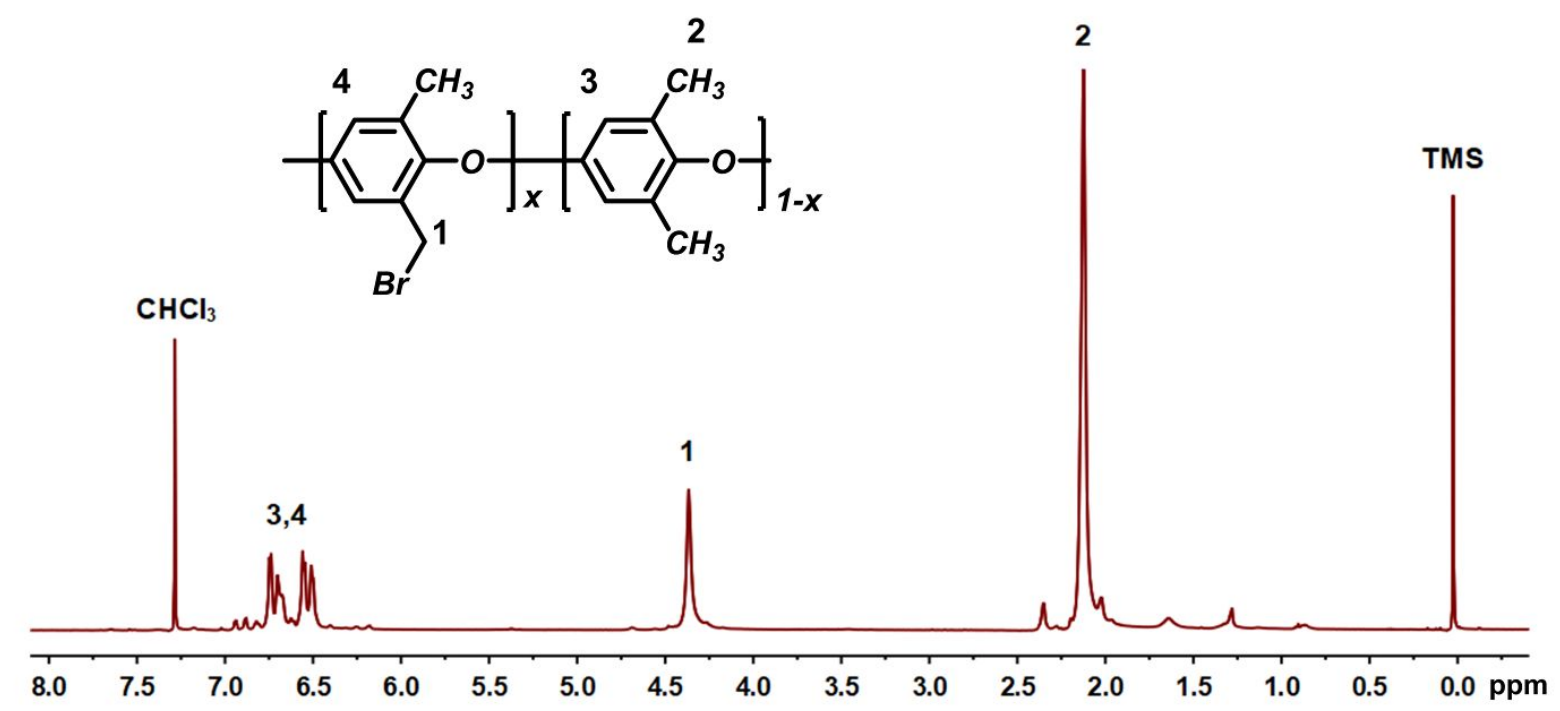

Figure S1. ${ }^{1} \mathrm{H}$ NMR spectra of BPPO (400 MHz, $\left.\mathrm{CDCl}_{3}, 298 \mathrm{~K}\right)$. 


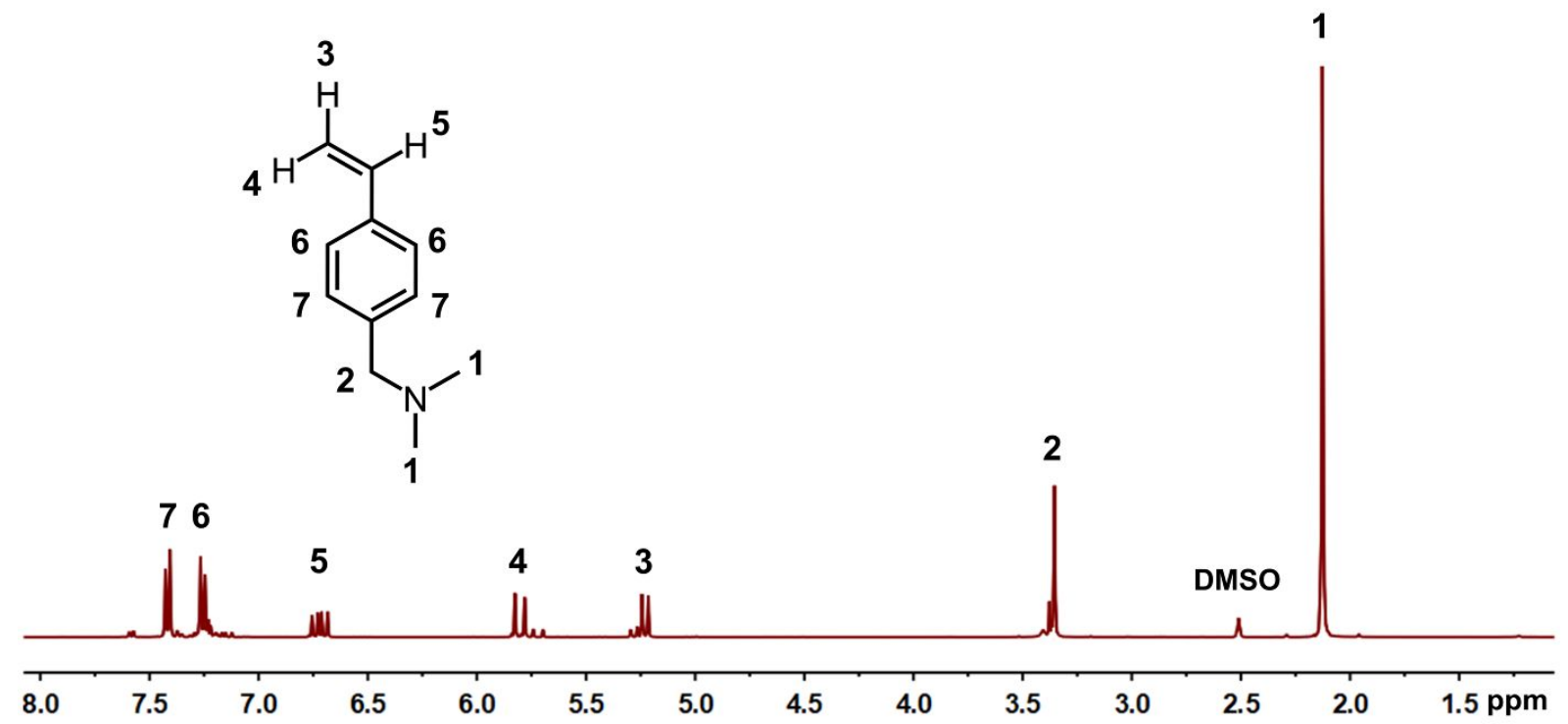

Figure S2. ${ }^{1} \mathrm{H}$ NMR spectrum of VBN $\left(400 \mathrm{MHz}\right.$, DMSO- $\left.d_{6}, 298 \mathrm{~K}\right)$. 


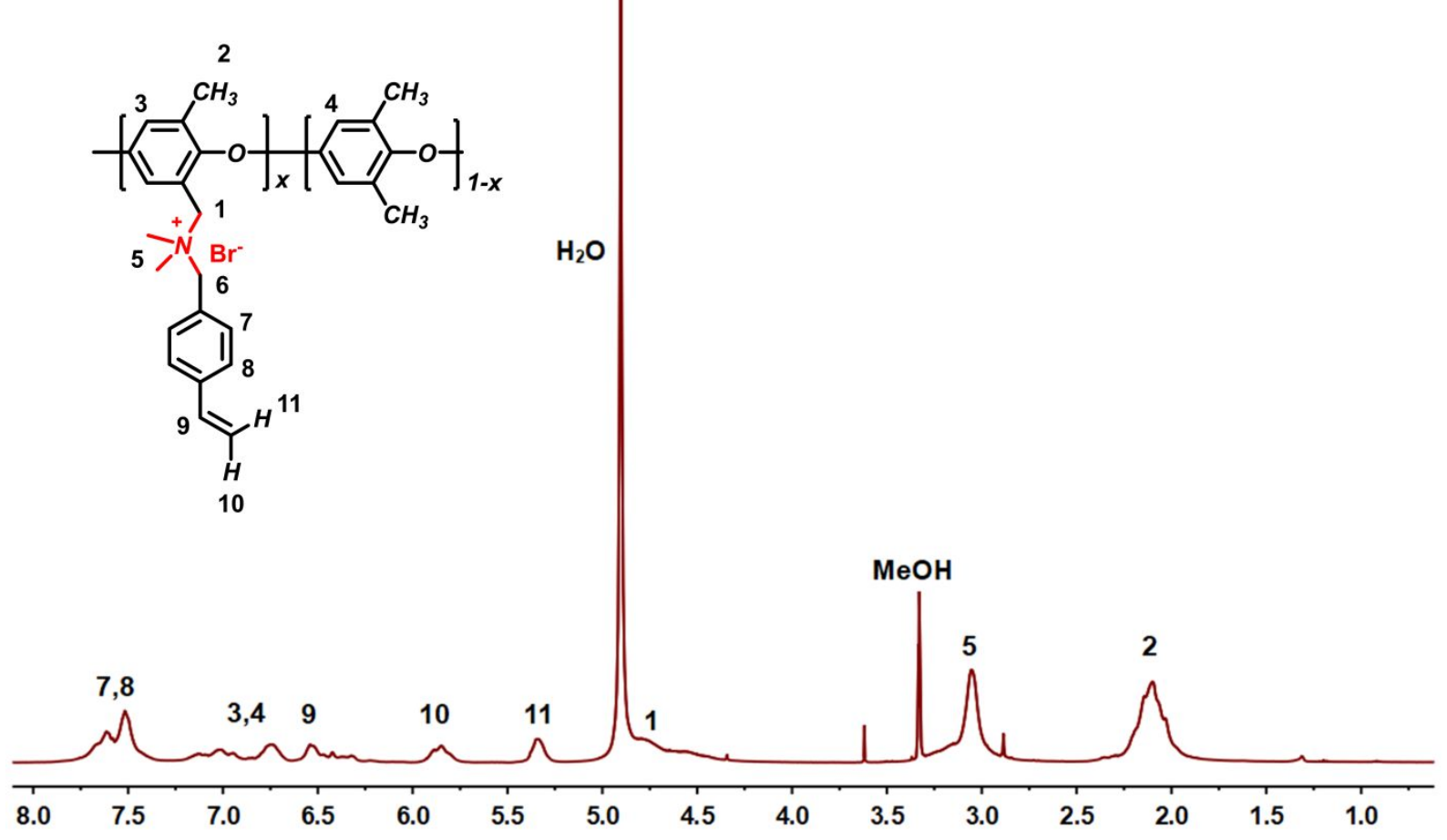

Figure S3. ${ }^{1} \mathrm{H}$ NMR spectrum of VBQPPO (400 MHz, $\left.\mathrm{CD}_{3} \mathrm{OD}, 298 \mathrm{~K}\right)$. 

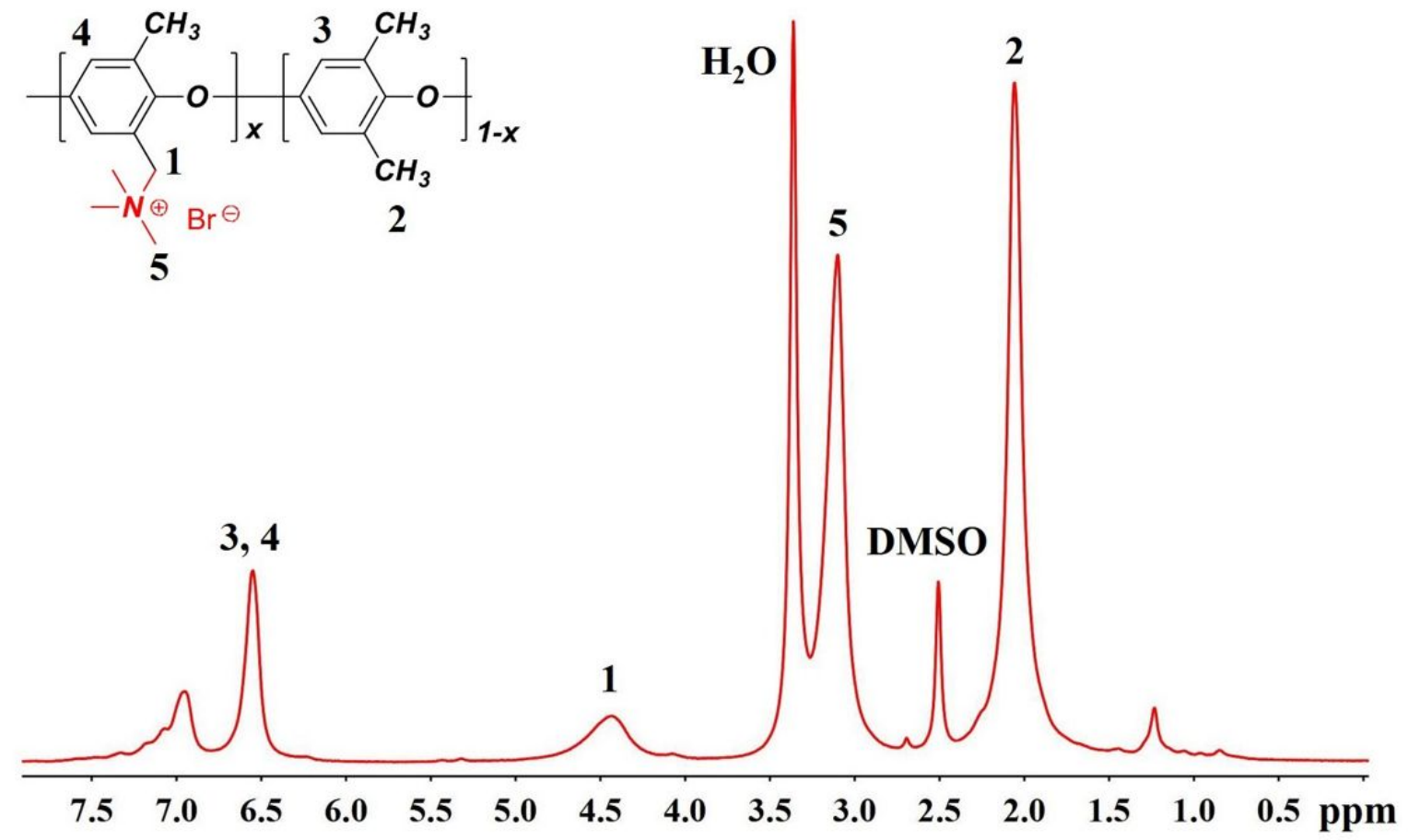

Figure S4. ${ }^{1} \mathrm{H}$ NMR spectrum of QPPO (400 MHz, $\left.\mathrm{CD}_{3} \mathrm{OD}, 298 \mathrm{~K}\right)$. 


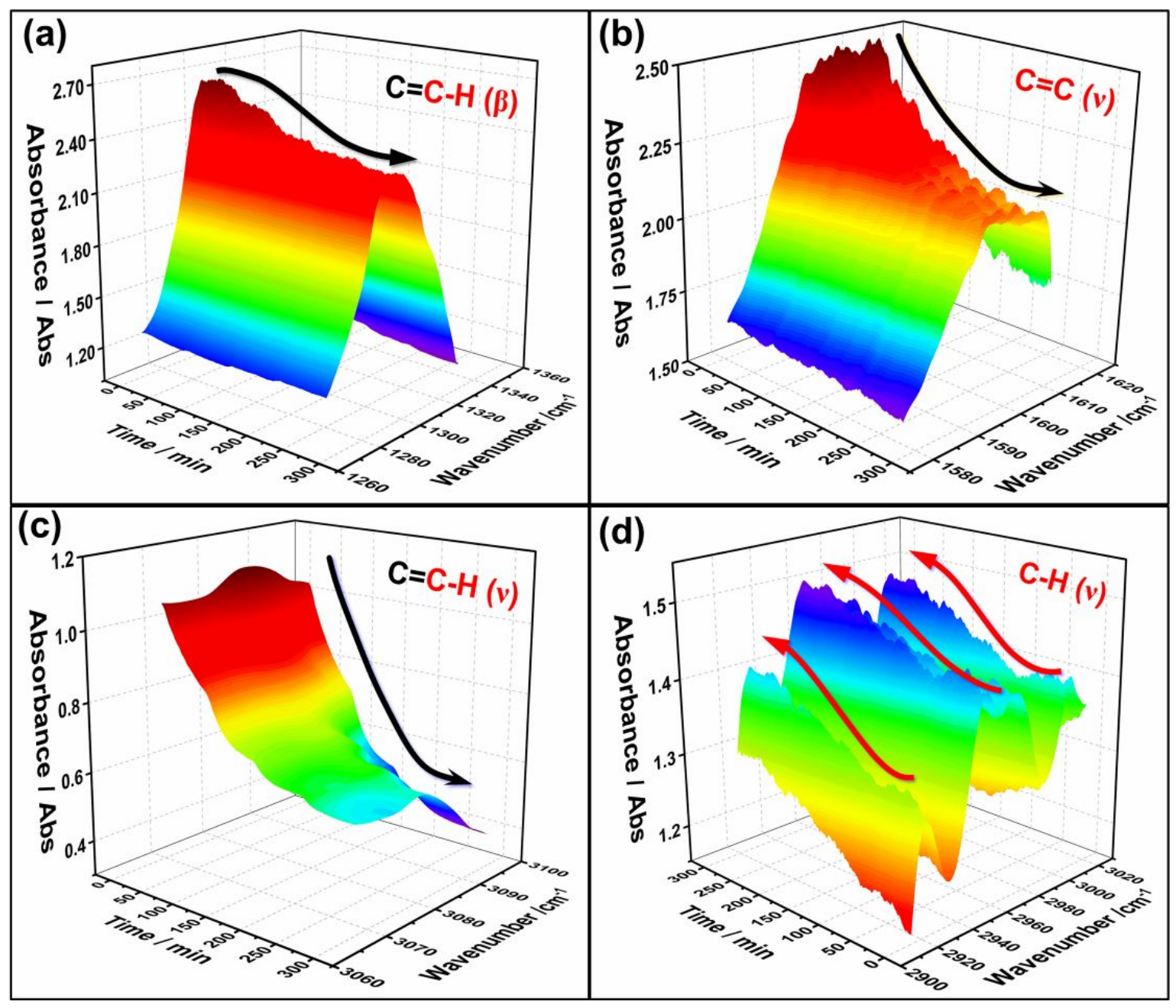

Figure S5. The change in in situ the FTIR spectra of VBQPPO (immobilized between two $\mathrm{CaF}_{2}$ optical plates) during heating from $30{ }^{\circ} \mathrm{C}$ to $70{ }^{\circ} \mathrm{C}$ in four stages: $40^{\circ} \mathrm{C}, 50{ }^{\circ} \mathrm{C}, 60{ }^{\circ} \mathrm{C}$, and $70{ }^{\circ} \mathrm{C}\left(10^{\circ} \mathrm{C}\right.$ temperature rise over 10 minutes followed by a thermal hold at each temperature of $60 \mathrm{~min}$ ). 


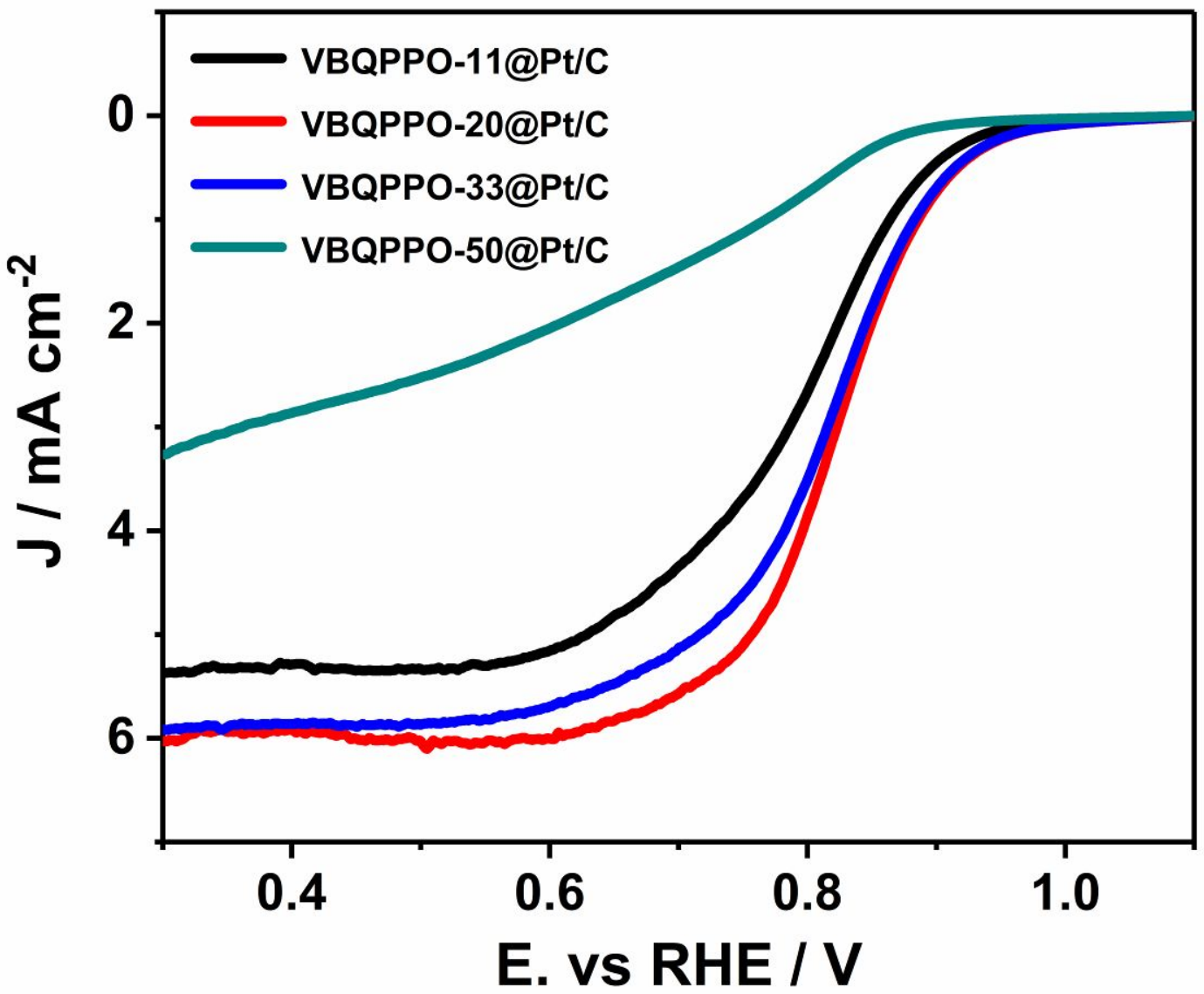

Figure S6. Linear scan voltammograms for Pt/C-based working electrodes (submerged in $\mathrm{O}_{2}$-purged aqueous $\mathrm{KOH}$ $(0.1 \mathrm{M})$ solution) containing various VBQPPO loadings (the numbers in the legend are the weight content of VBQPPO). 


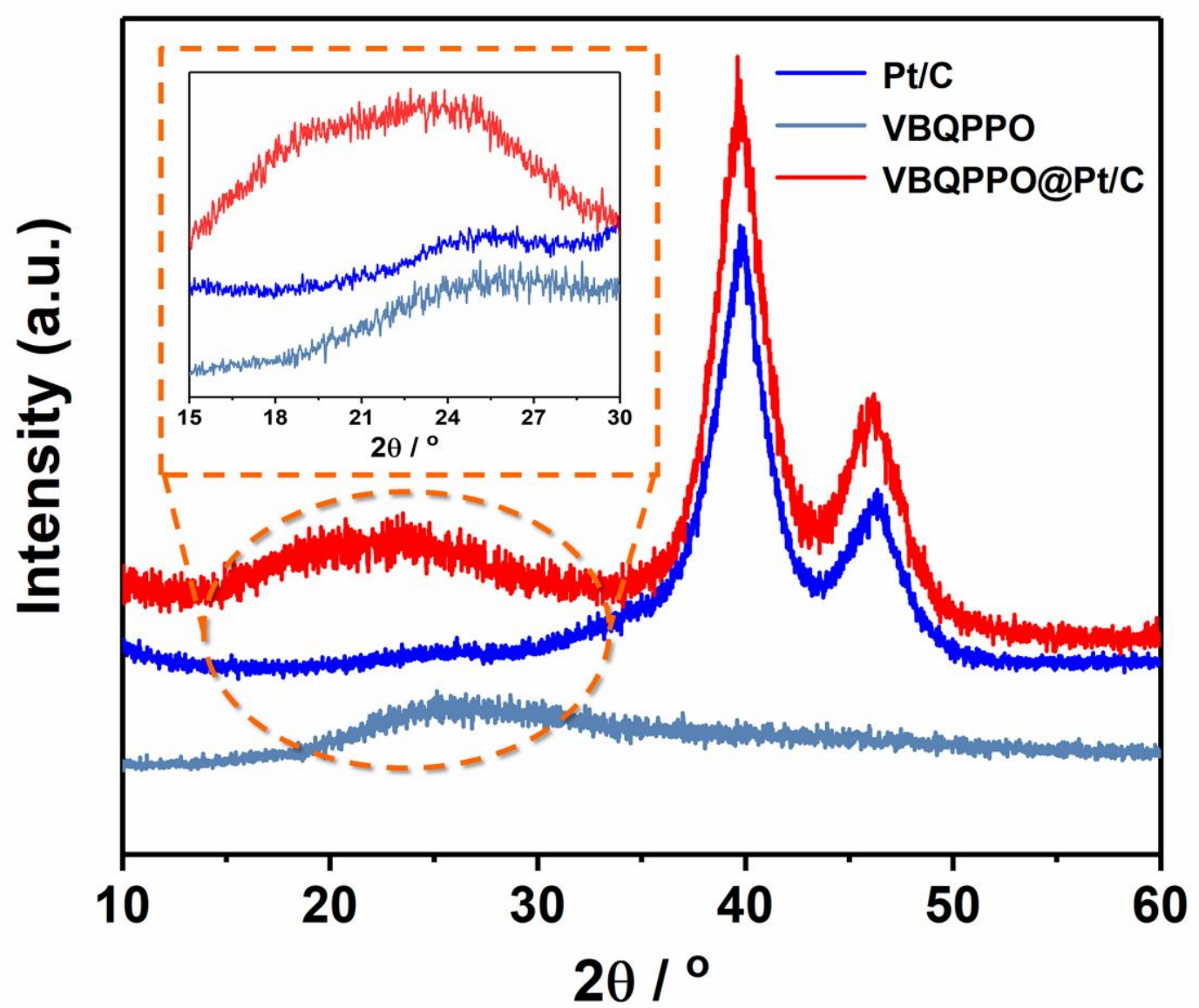

Figure S7. Powder XRD diffraction patterns of the Pt/C catalyst encapsulated by the VBQPPO ionomer. 


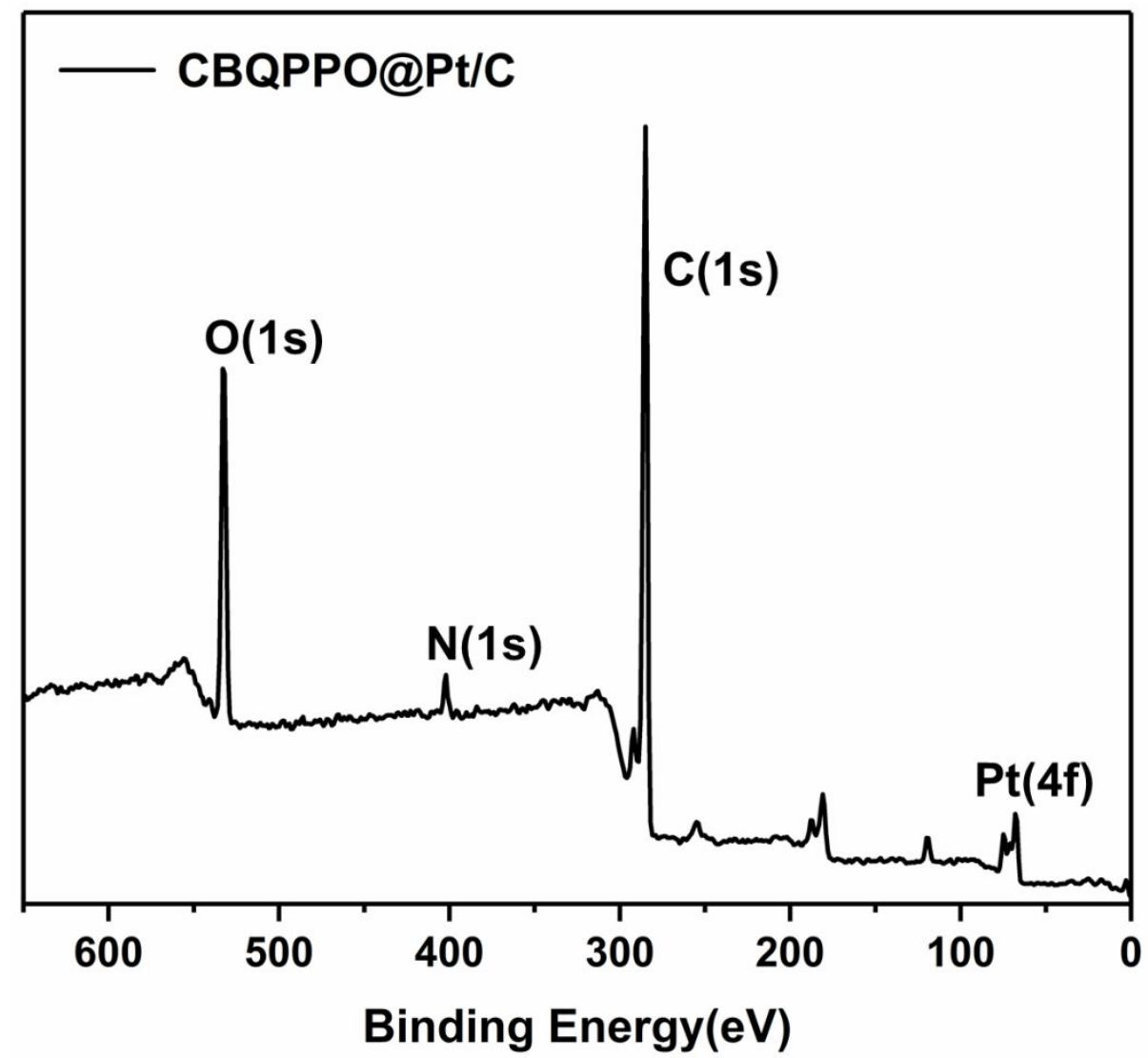

Figure S8. Powder XPS spectra of the Pt/C catalyst encapsulated by the CBQPPO ionomer. ${ }^{16}$ 

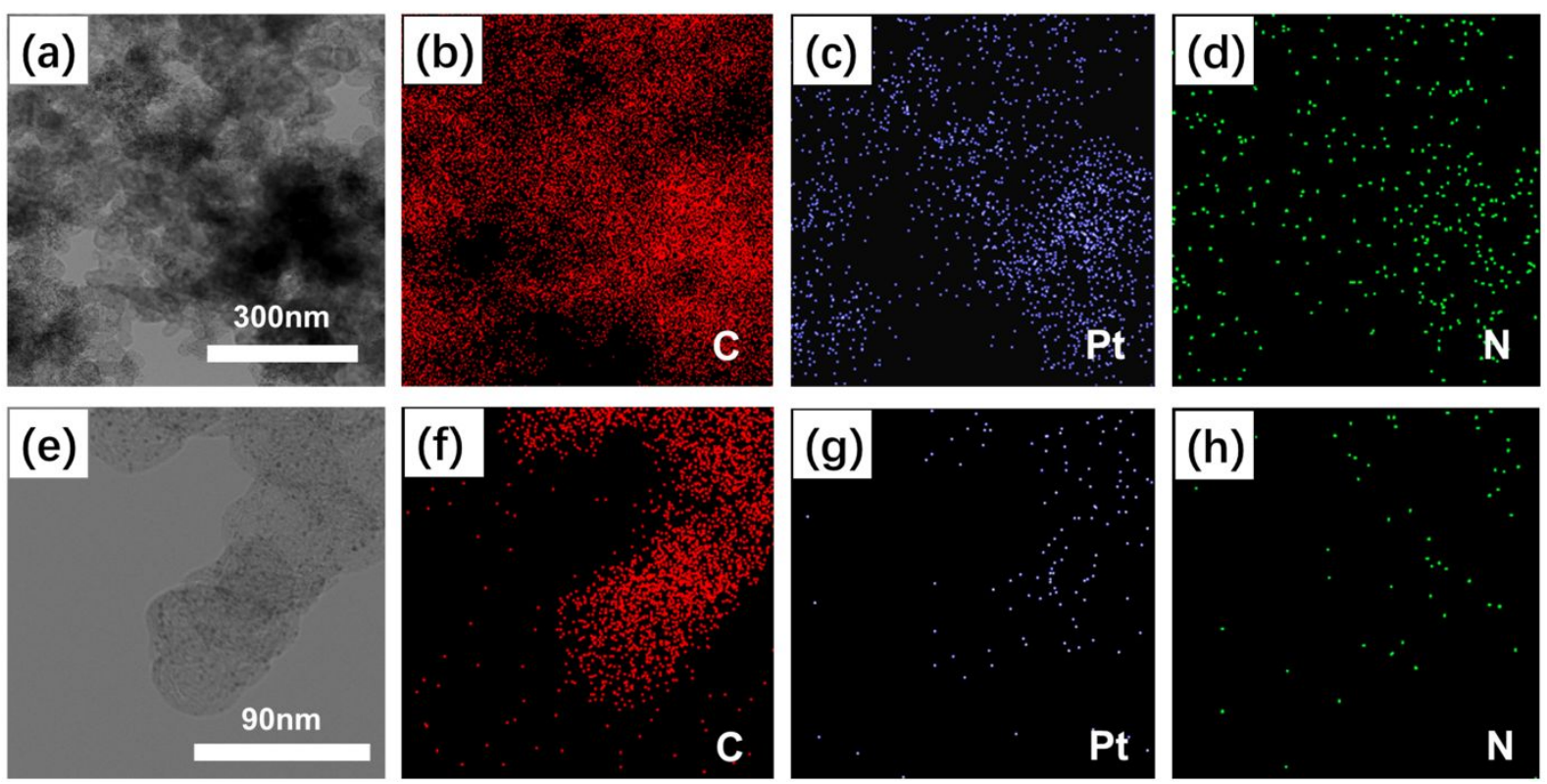

Figure S9. TEM and relative mapping of the Pt/C catalyst encapsulated by the CBQPPO ionomer. 


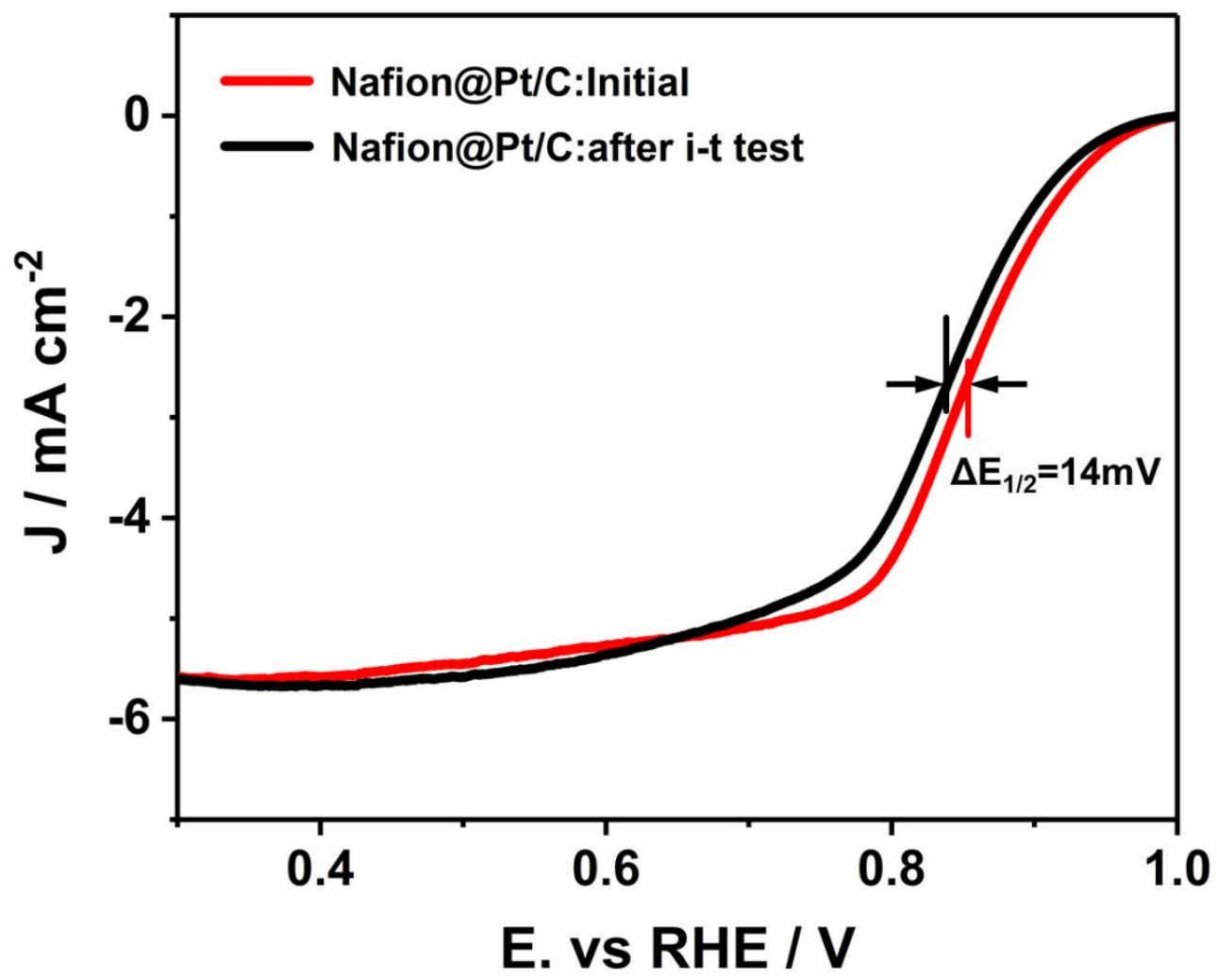

Figure S10. Linear scan voltammograms in $\mathrm{O}_{2}$-saturated aqueous $\mathrm{KOH}(0.1 \mathrm{M})$ solution (room temperature with a sweep rate of $10 \mathrm{mV} \mathrm{S}^{-1}$ and a rotation rate of $1600 \mathrm{rpm}$ ) of the nafion@Pt/C cathode catalysts before (red) and after (black) chronoamperometry (i-t) test (potential hold at $-0.50 \mathrm{~V}$ vs. $\mathrm{Ag} / \mathrm{AgCl}$ reference electrode (0.498 V vs. RHE) for $250 \mathrm{~min})$. 


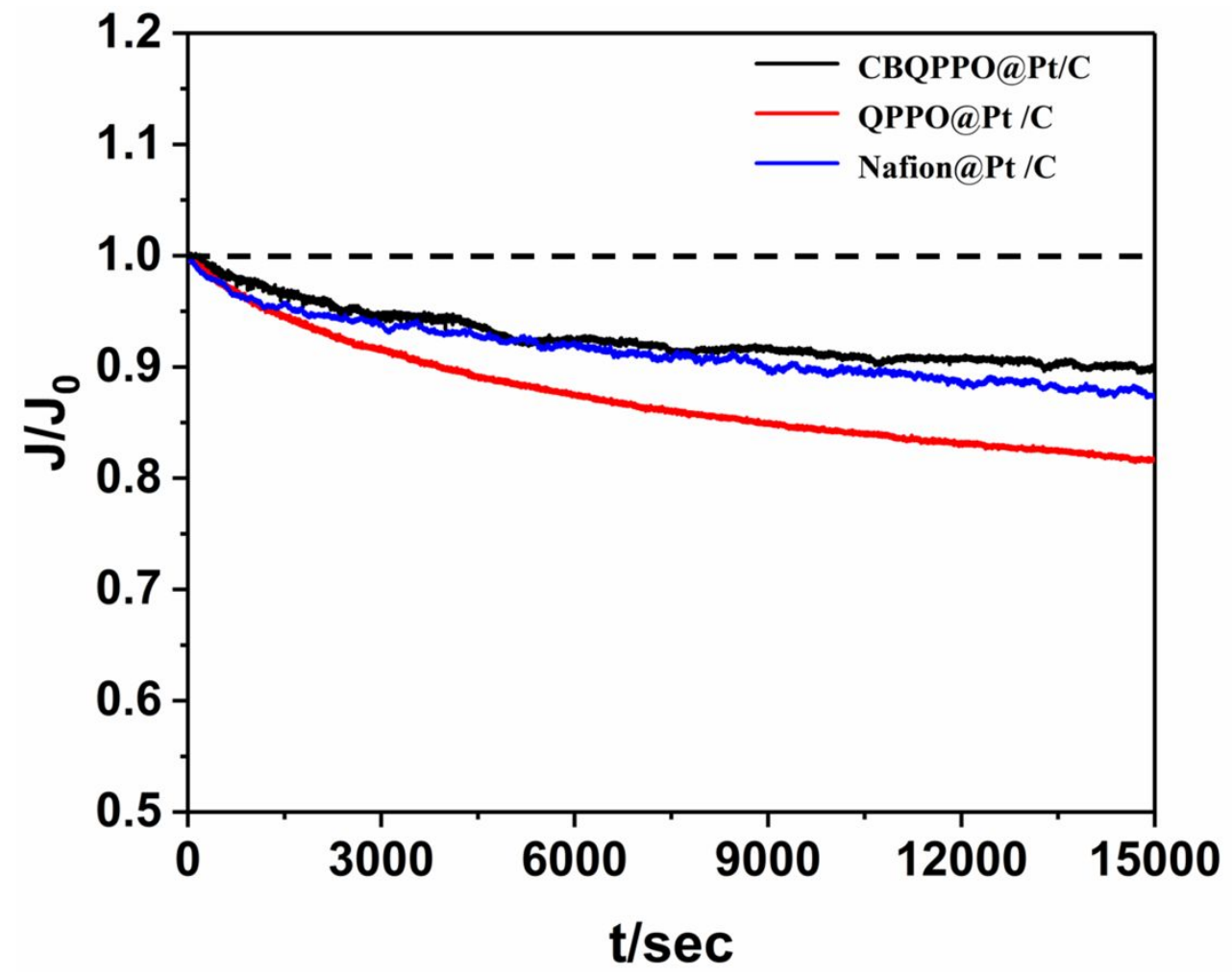

Figure S11. Current vs. time $(i-t)$ chronoamperometric responses of CBQPPO@Pt/C, QPPO@Pt/C and nafion@Pt/C working electrodes poised at $-0.5 \mathrm{~V}$ vs. $\mathrm{Ag} / \mathrm{AgCl}\left(0.498 \mathrm{~V} v\right.$ s. RHE) in $\mathrm{O}_{2}$-purged aqueous $\mathrm{KOH}(0.1 \mathrm{M})$. 


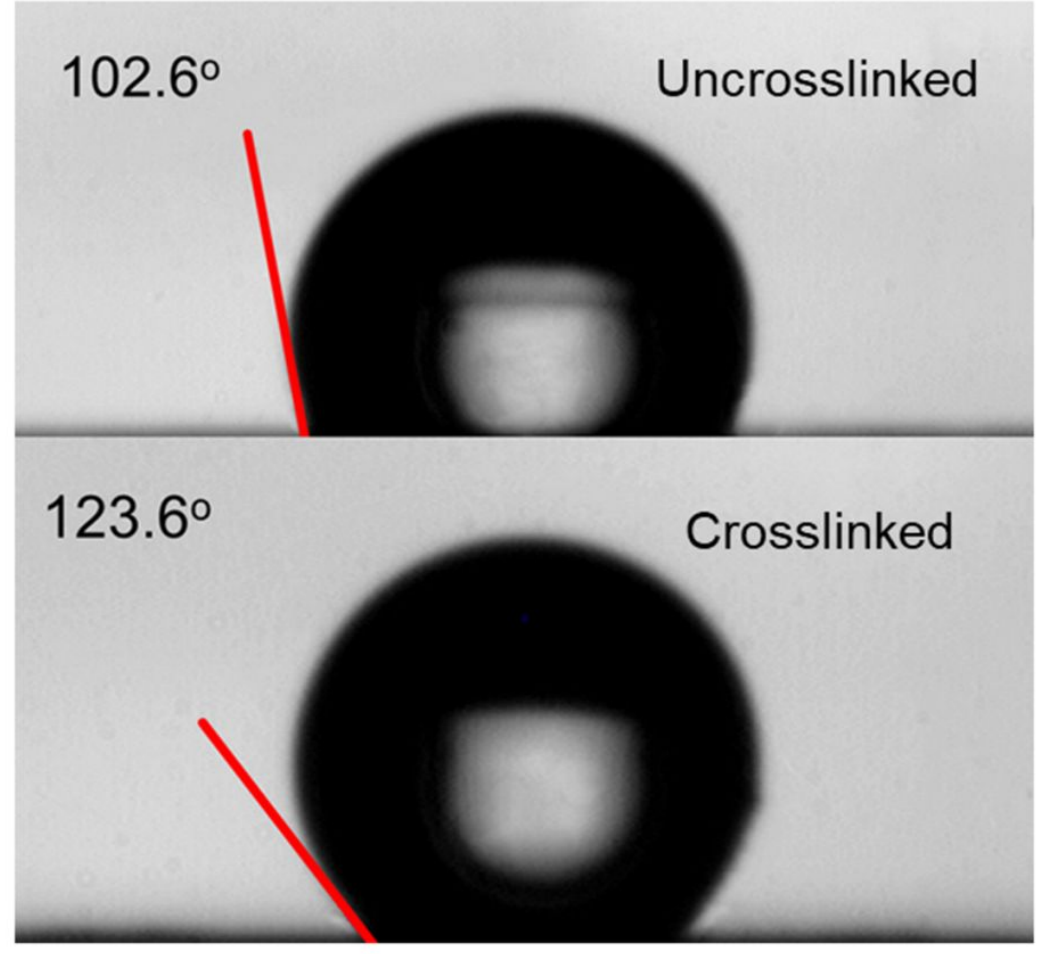

Figure S12. Static water contact angle at the surfaces of catalyst layers containing QPPO@Pt/C-GDE and CBQPPO@Pt/C gas diffusion electrodes (GDE). 


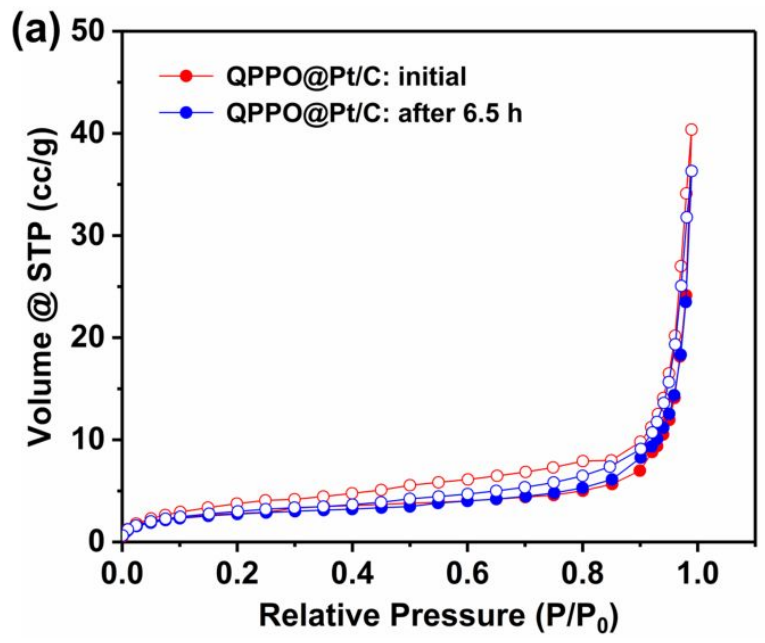

(c)
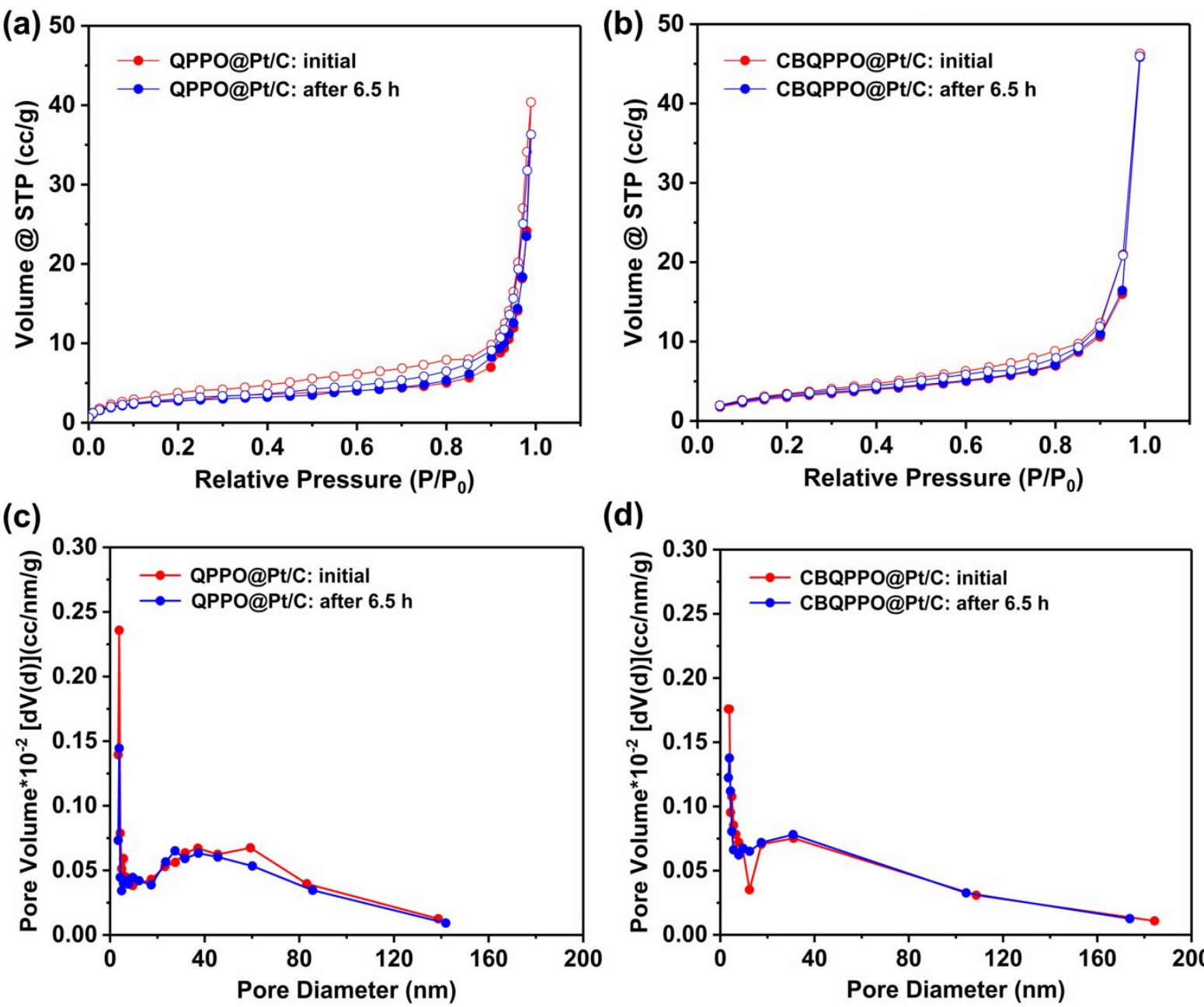

(d)

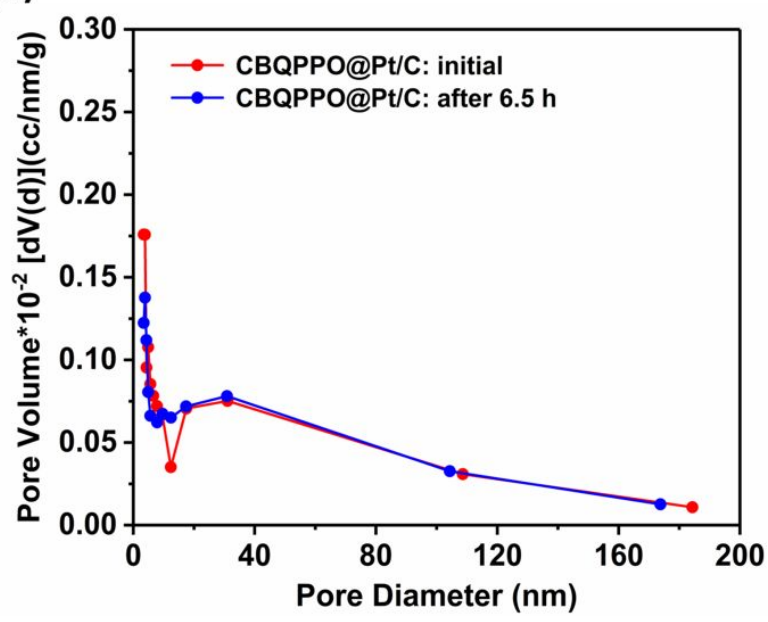

Figure S13. $\mathrm{N}_{2}$ adsorption/desorption isotherm before and after the durability test: (a) QPPO@Pt/C-based MEAs, (b)CBQPPO@Pt/C-based MEAs. Pore size distributions before and after the durability test: (c) QPPO@Pt/C-based MEAs, (d) CBQPPO@Pt/C-based MEAs. 


\section{References}

(1) Peng, H.; Li, Q.; Hu, M.; Xiao, L.; Lu, J.; Zhuang, L. Alkaline Polymer Electrolyte Fuel Cells Stably Working at $80^{\circ} \mathrm{C}$. J. Power Sources 2018, 390, 165-167.

(2) Wang, Y.; Yang, Y.; Jia, S.; Wang, X.; Lyu, K.; Peng, Y.; Zheng, H.; Wei, X.; Ren, H.; Xiao, L.; Wang, J.; Muller, D. A.; Abruna, H. D.; Hwang, B. J.; Lu, J.; Zhuang, L. Synergistic Mn-Co Catalyst Outperforms Pt on High-Rate Oxygen Reduction for Alkaline Polymer Electrolyte Fuel Cells. Nat. Commun. 2019, 10, 1506.

(3) Li, Q.; Peng, H.; Wang, Y.; Xiao, L.; Lu, J.; Zhuang, L. The Comparability of Pt to Pt-Ru in Catalyzing the Hydrogen Oxidation Reaction for Alkaline Polymer Electrolyte Fuel Cells Operated at $80^{\circ} \mathrm{C}$. Angew. Chem. Int. Ed. Engl. 2019, 58, 1442-1446.

(4) Peng, X.; Kashyap, V.; Ng, B.; Kurungot, S.; Wang, L.; Varcoe, J. R.; Mustain, W. E. High-Performing PGM-Free AEMFC Cathodes from Carbon-Supported Cobalt Ferrite Nanoparticles. Catalysts 2019, 9, 264.

(5) Omasta, T. J.; Wang, L.; Peng, X.; Lewis, C. A.; Varcoe, J. R.; Mustain, W. E. Importance of Balancing Membrane and Electrode Water in Anion Exchange Membrane Fuel Cells. J. Power Sources 2018, 375, 205-213.

(6) Omasta, T. J.; Peng, X.; Miller H. A.; Vizza, F.; Wang, L.; Varcoe, J. R.; Dekel, D. R.; Mustain, W. E. Beyond $1.0 \mathrm{~W} \mathrm{~cm}^{-2}$ Performance without Platinum: The Beginning of a New Era in Anion Exchange Membrane Fuel Cells. J. electrochem. Soc. 2018, 165, J3039-J3044.

(7) Omasta, T. J.; Park, A. M.; LaManna, J. M.; Zhang, Y.; Peng, X.; Wang, L.; Jacobson, D. L.; Varcoe, J. R.; Hussey, D. S.; Pivovar, B. S.; Mustain, W. E. Beyond Catalysis and Membranes: Visualizing and Solving the Challenge of Electrode Water Accumulation and Flooding in AEMFCs. Energy Environ. Sci. 2018, 11, 551-558.

(8) Wang, L.; Bellini, M.; Miller, H. A.; Varcoe, J. R. A High Conductivity Ultrathin Anion-Exchange Membrane with 500+ h Alkali Stability for Use in Alkaline Membrane Fuel Cells that can Achieve $2 \mathrm{~W} \mathrm{~cm}^{-2}$ at $80^{\circ} \mathrm{C}$. J. Mater. Chem. A 2018, 6, 1540415412.

(9) Wang, L.; Brink, J. J.; Liu, Y.; Herring, A. M.; Ponce-González, J.; Whelligan, D. K.; Varcoe, J. R. Non-fluorinated PreIrradiation-Grafted (Peroxidated) LDPE-Based Anion-Exchange Membranes with High Performance and Stability. Energy Environ. Sci. 2017, 10, 2154-2167.

(10) Wang, L.; Magliocca, E.; Cunningham, E. L.; Mustain, W. E.; Poynton, S. D.; Escudero-Cid, R.; Nasef, M. M.; Ponce-Gonzalez, J.; Bance-Souahli, R.; Slade, R. C. T.; Whelligan, D. K.; and Varcoe, J. R. An Optimised Synthesis of High Performance RadiationGrafted Anion-Exchange Membranes. Green Chem. 2017, 19, 831-843.

(11) Wang, L.; Peng, X.; Mustain, W. E.; Varcoe, J. R. Radiation-Grafted Anion-Exchange Membranes: The Switch from Low- to High-Density Polyethylene Leads to Remarkably Enhanced Fuel Cell Performance. Energy Environ. Sci. 2019, 12, 1575-1579.

(12) Gonçalves Biancolli, A. L.; Herranz, D.; Wang, L.; Stehlíková, G.; Bance-Soualhi, R.; Ponce-González, J.; Ocón, P.; Ticianelli, E. A.; Whelligan, D. K.; Varcoe, J. R.; Santiago, E. I. ETFE-Based Anion-Exchange Membrane Ionomer Powders for Alkaline Membrane Fuel Cells: A First Performance Comparison of Head-Group Chemistry. J. Mater. Chem. A 2018, 6, 24330-24341.

(13) Ponce-González, J.; Ouachan, I.; Varcoe, J. R.; Whelligan, D. K. Radiation-Induced Grafting of a Butyl-Spacer Styrenic Monomer onto ETFE: The Synthesis of the Most Alkali Stable Radiation-Grafted Anion-Exchange Membrane to Date. J. Mater. Chem. A 2018, 6, 823-827.

(14) Maurya, S.; Noh, S.; Matanovic, I.; Park, E. J.; Narvaez Villarrubia, C.; Martinez, U.; Han, J.; Bae, C.; Kim, Y. S., Rational Design of Polyaromatic Ionomers for Alkaline Membrane Fuel Cells with $>1 \mathrm{~W} \mathrm{~cm}^{-2}$ Power Density. Energy Environ. Sci. 2018, 11, 3283-3291.

(15) Huang, G.; Mandal, M.; Peng, X.; Yang-Neyerlin, A. C.; Pivovar, B. S.; Mustain, W. E.; Kohl, P. A. Composite Poly(norbornene) Anion Conducting Membranes for Achieving Durability, Water Management and High Power $\left(3.4 \mathrm{~W} / \mathrm{cm}^{2}\right)$ in Hydrogen/Oxygen Alkaline Fuel Cells. J. Electrochem. Soc. 2019, 166, F637-F644.

(16) Moulder, J. F.; Stickle, W. F.; Sobol, P. E.; Bomben, K. D. Handbook of X-ray Photoelectron Spectroscopy; Chastain, J., Ed.; Perkin-Elmer Corporation, 1992. 